\title{
Matrix representations of finitely generated Grassmann algebras and some consequences
}

\author{
László Márki, Johan Meyer, Jenő Szigeti, and Leon van Wyk
}

\begin{abstract}
We prove that the $m$-generated Grassmann algebra can be embedded into a $2^{m-1} \times 2^{m-1}$ matrix algebra over a factor of a commutative polynomial algebra in $m$ indeterminates. Cayley-Hamilton and standard identities for $n \times n$ matrices over the $m$-generated Grassmann algebra are derived from this embedding. Other related embedding results are also presented.
\end{abstract}

\section{INTRODUCTION}

Let $K$ be a field (of characteristic zero) and consider the free associative $K$ algebra $K\left\langle x_{1}, \ldots, x_{m}\right\rangle$ generated by the (non-commuting) indeterminates $x_{1}, \ldots, x_{m}$. The elements of $K\left\langle x_{1}, \ldots, x_{m}\right\rangle$ are $K$-linear combinations of monomials of the form $x_{i_{1}} \cdots x_{i_{k}}$ with $i_{1}, \ldots, i_{k} \in\{1, \ldots, m\}$ (not necessarily different). The $m$-generated Grassmann (or exterior) algebra is defined as the factor $K$-algebra

$$
E^{(m)}=K\left\langle x_{1}, \ldots, x_{m}\right\rangle / I\left(x_{1}, \ldots, x_{m}\right),
$$

where

$$
I\left(x_{1}, \ldots, x_{m}\right)=\left(x_{i} x_{j}+x_{j} x_{i} \mid 1 \leq i \leq j \leq m\right)_{K}
$$

is the (two-sided) $K$-ideal of $K\left\langle x_{1}, \ldots, x_{m}\right\rangle$ generated by the polynomials $x_{i} x_{j}+$ $x_{j} x_{i}$ for $1 \leq i \leq j \leq m$. The usual notation for this Grassmann algebra is

$$
\left.E^{(m)}=K\left\langle v_{1}, \ldots, v_{m}\right| v_{i} v_{j}+v_{j} v_{i}=0 \text { for all } 1 \leq i \leq j \leq m\right\rangle,
$$

where the cosets $v_{i}=x_{i}+I\left(x_{1}, \ldots, x_{m}\right)$ are the anticommuting generators of $E^{(m)}$. Clearly, any element of $E^{(m)}$ is a unique $K$-linear combination of monomials of the form $v_{i_{1}} \cdots v_{i_{k}}$ with $1 \leq i_{1}<\cdots<i_{k} \leq m$. It follows that $\operatorname{dim}_{K} E^{(m)}=2^{m}$.

1991 Mathematics Subject Classification. 15A75, 16G30, 16R10.

Key words and phrases. Grassmann algebra, matrix algebra, $K$-embedding, CayleyHamilton identity, skew polynomial algebra.

The first and third named authors were supported by OTKA K-101515 of Hungary.

The second and fourth named authors were supported by the National Research Foundation of South Africa under Grant No. UID 72375. Any opinion, findings and conclusions or recommendations expressed in this material are those of the authors and therefore the National Research Foundation does not accept any liability in regard thereto.

As far as the third author is concerned, this research was partly carried out as part of the TAMOP-4.2.1.B-10/2/KONV-2010-0001 project with support from the European Union, cofinanced by the European Social Fund.

The authors thank P. N. Ánh for fruitful discussions. 
The definition of the countably infinitely generated Grassmann algebra

$$
\left.E=K\left\langle v_{1}, \ldots, v_{m}, \ldots\right| v_{i} v_{j}+v_{j} v_{i}=0 \text { for all } 1 \leq i \leq j\right\rangle
$$

is similar.

For a ring (or $K$-algebra) $R$ let $\mathrm{M}_{n}(R)$ denote the full $n \times n$ matrix ring $(K$ algebra) over $R$ with identity $I_{n} \in \mathrm{M}_{n}(R)$. Since any matrix $A \in \mathrm{M}_{n}(E)$ has a finite number of entries and each entry contains a finite number of generators $v_{i}$ from $E$, there exists an integer $m \geq 1$ such that $A \in \mathrm{M}_{n}\left(E^{(m)}\right)$. It follows that

$$
\mathrm{M}_{n}(E)=\bigcup_{m=1}^{\infty} \mathrm{M}_{n}\left(E^{(m)}\right) .
$$

The algebras $E^{(m)}$ and $E$ play a fundamental role in many areas of mathematics.

Our main inspiration is Kemer's pioneering work $[\mathrm{K}]$ on the $T$-ideals of associative algebras, which revealed the importance of the identities satisfied by the full $n \times n$ matrix algebra $\mathrm{M}_{n}(E)$ and by the algebra $\mathrm{M}_{n, t}(E)$ of $(n, t)$-supermatrices (this is a certain $K$-subalgebra of $\left.\mathrm{M}_{n}(E)\right)$. The prime $T$-ideals of $K\left\langle x_{1}, \ldots, x_{m}, \ldots\right\rangle$ are exactly the $T$-ideals of the identities satisfied by $\mathrm{M}_{n}(K)$ for $n \geq 1$. The $T$-prime $T$-ideals are the prime $T$-ideals plus the $T$-ideals of the identities of $\mathrm{M}_{n}(E)$ for $n \geq 1$ and of $\mathrm{M}_{n, t}(E)$ for $n-1 \geq t \geq 1$. Another remarkable result is that, for $n$ sufficiently large, any $T$-ideal contains the $T$-ideal of the identities satisfied by $\mathrm{M}_{n}(E)$. Thus the algebras $\mathrm{M}_{n}(E)$ and $\mathrm{M}_{n}\left(E^{(m)}\right)$ served as the main motivation for the present work.

An additional motivation is the well-known embedding of the skew field $\mathbb{H}$ of the real quaternions into $4 \times 4$ real matrices:

$$
a+b i+c j+d k \longmapsto\left[\begin{array}{rrrr}
a & b & c & d \\
-b & a & -d & c \\
-c & d & a & -b \\
-d & -c & b & a
\end{array}\right],
$$

where $a, b, c, d \in \mathbb{R}$. The above definition provides an injective $\mathbb{R}$-algebra homomorphism $v: \mathbb{H} \rightarrow \mathrm{M}_{4}(\mathbb{R})$. Using the natural extension

$$
v_{n}: \mathrm{M}_{n}(\mathbb{H}) \longrightarrow \mathrm{M}_{n}\left(\mathrm{M}_{4}(\mathbb{R})\right) \cong \mathrm{M}_{4 n}(\mathbb{R}),
$$

an $n \times n$ matrix over $\mathbb{H}$ can be viewed as a $4 n \times 4 n$ matrix over $\mathbb{R}$. For a quaternionic matrix $A \in \mathrm{M}_{n}(\mathbb{H})$, the Cayley-Hamilton identity for $v_{n}(A)$ yields the same identity (with real coefficients) of degree $4 n$ for $A$ itself.

A similar approach to get a Cayley-Hamilton identity of degree $2 n$ for a matrix $A \in \mathrm{M}_{n}\left(E^{(2)}\right)$ is based on embedding the two-generated exterior algebra $E^{(2)}$ into a $2 \times 2$ matrix algebra over a certain commutative ring (see [SzvW]).

In order to present a Cayley-Hamilton identity of degree $2^{m-1} n$ for a matrix in $\mathrm{M}_{n}\left(E^{(m)}\right)$, first we consider the so called constant trace (CT-)representations of an arbitrary $K$-algebra. In Section 2 we derive a Cayley-Hamilton identity for $n \times n$ matrices over any algebra having a CT-representation. Then in Section 3, using induction, we present a CT-representation ( $K$-embedding)

$$
\varepsilon^{(m)}: E^{(m)} \longrightarrow \mathrm{M}_{2^{m-1}}\left(K\left[z_{1}, \ldots, z_{m}\right] /\left(z_{1}^{2}, \ldots, z_{m}^{2}\right)\right) .
$$

Notice that we cannot expect similar results for the infinitely generated Grassmann algebra $E$. Since $E$ does not satisfy any of the standard identities, it follows that $E$ does not embed into any full matrix algebra over a commutative ring. 
Finally in Section 4 we use a certain factor of a skew polynomial algebra to give a broad generalization of the embedding process for $E^{(m)}$.

\section{CT-REPRESENTATIONS AND CAYLEY-HAMILTON IDENTITIES}

Let ${ }_{K} R$ be an arbitrary and ${ }_{K} \Omega$ be a commutative (associative) algebra over $K$ (notice that $K \subseteq \mathrm{Z}(R)$ and $K \subseteq \Omega$ ). For an integer $t \geq 1$, we consider representations of $R$ over $\Omega$ which are injective $K$-algebra homomorphisms ( $K$-embeddings) $\varepsilon: R \longrightarrow \mathrm{M}_{t}(\Omega)$. We call $\varepsilon$ a constant trace (CT-) representation if $\operatorname{tr}(\varepsilon(r)) \in K$ for all $r \in R$ (here $\operatorname{tr}(\varepsilon(r))$ is the sum of the diagonal entries of the $t \times t$ matrix $\left.\varepsilon(r) \in \mathrm{M}_{t}(\Omega)\right)$.

2.1.Theorem. Let $\varepsilon: R \longrightarrow \mathrm{M}_{t}(\Omega)$ be a CT-representation of $R$ over $\Omega$. If $A \in \mathrm{M}_{n}(R)$ is an $n \times n$ matrix, then $A$ satisfies a Cayley-Hamilton identity of the form

$$
A^{t n}+c_{1} A^{t n-1}+\cdots+c_{t n-1} A+c_{t n} I_{n}=0,
$$

where $c_{i} \in K, 1 \leq i \leq t n$.

Proof. Let

$$
\varepsilon_{n}: \mathrm{M}_{n}(R) \longrightarrow \mathrm{M}_{n}\left(\mathrm{M}_{t}(\Omega)\right) \cong \mathrm{M}_{t n}(\Omega)
$$

be the natural extension of $\varepsilon$. For any matrix $A=\left[a_{i, j}\right]$ in $\mathrm{M}_{n}(R)$, the trace of the $t n \times t n$ matrix $B=\varepsilon_{n}(A)$ is the sum of the traces of the diagonal $t \times t$ blocks:

$$
\operatorname{tr}(B)=\sum_{i=1}^{n} \operatorname{tr}\left(\varepsilon\left(a_{i, i}\right)\right)
$$

Since $\varepsilon$ is a CT-representation, we have $\operatorname{tr}\left(\varepsilon\left(a_{i, i}\right)\right) \in K$ for each $1 \leq i \leq n$. It follows that $\operatorname{tr}(B) \in K$. For the coefficients of the characteristic polynomial

$$
\operatorname{det}(z I-B)=c_{0} z^{t n}+c_{1} z^{t n-1}+\cdots+c_{t n-1} z+c_{t n} \in \Omega[z]
$$

of $B$, the following recursion holds: $c_{0}=1$ and

$$
c_{k}=-\frac{1}{k}\left(c_{k-1} \operatorname{tr}(B)+c_{k-2} \operatorname{tr}\left(B^{2}\right)+\cdots+c_{1} \operatorname{tr}\left(B^{k-1}\right)+c_{0} \operatorname{tr}\left(B^{k}\right)\right)
$$

for $1 \leq k \leq t n$ (Newton formulae, see $[\mathrm{R}]$ ). In view of

$$
\operatorname{tr}\left(B^{k}\right)=\operatorname{tr}\left(\left(\varepsilon_{n}(A)\right)^{k}\right)=\operatorname{tr}\left(\varepsilon_{n}\left(A^{k}\right)\right) \in K,
$$

we deduce that $c_{i} \in K$ for each $0 \leq i \leq t n$. Thus $\operatorname{det}(z I-B) \in K[z]$ and the Cayley-Hamilton identity for $B \in \mathrm{M}_{t n}(\Omega)$ is of the form

$$
B^{t n}+c_{1} B^{t n-1}+\cdots+c_{t n-1} B+c_{t n} I_{n}=0 .
$$

It follows that

$$
\begin{gathered}
\left(\varepsilon_{n}(A)\right)^{t n}+c_{1}\left(\varepsilon_{n}(A)\right)^{t n-1}+\cdots+c_{t n-1} \varepsilon_{n}(A)+c_{t n} I_{n}= \\
\varepsilon_{n}\left(A^{t n}+c_{1} A^{t n-1}+\cdots+c_{t n-1} A+c_{t n} I_{n}\right)=0
\end{gathered}
$$

holds in $\mathrm{M}_{t n}(\Omega)$, thus the injectivity of $\varepsilon_{n}$ gives the desired identity. 


\section{CT-REPRESENTATION OF $E^{(m)}$}

For $m=1$ we have a natural isomorphism $E^{(1)}=K\left\langle v_{1} \mid v_{1}^{2}=0\right\rangle \cong K[z] /\left(z^{2}\right)$ of $K$-algebras. If $m=2$, then the assignments

$$
1 \longmapsto\left[\begin{array}{cc}
1 & 0 \\
0 & 1
\end{array}\right], v_{1} \longmapsto\left[\begin{array}{cc}
z_{1} & 0 \\
0 & -z_{1}
\end{array}\right], v_{2} \longmapsto\left[\begin{array}{cc}
0 & z_{2} \\
z_{2} & 0
\end{array}\right]
$$

define the following CT-representation $\varepsilon^{(2)}: E^{(2)} \longrightarrow \mathrm{M}_{2}\left(K\left[z_{1}, z_{2}\right] /\left(z_{1}^{2}, z_{2}^{2}\right)\right)$ :

$\varepsilon^{(2)}\left(c_{0}+c_{1} v_{1}+c_{2} v_{2}+c_{3} v_{1} v_{2}\right)=\left[\begin{array}{cc}c_{0}+c_{1} z_{1}+\left(z_{1}^{2}, z_{2}^{2}\right) & c_{2} z_{2}+c_{3} z_{1} z_{2}+\left(z_{1}^{2}, z_{2}^{2}\right) \\ c_{2} z_{2}-c_{3} z_{1} z_{2}+\left(z_{1}^{2}, z_{2}^{2}\right) & c_{0}-c_{1} z_{1}+\left(z_{1}^{2}, z_{2}^{2}\right)\end{array}\right]$,

where $c_{0}, c_{1}, c_{2}, c_{3} \in K$ and $\left(z_{1}^{2}, z_{2}^{2}\right)$ is the ideal of the commutative polynomial ring $K\left[z_{1}, z_{2}\right]$ generated by the monomials $z_{1}^{2}, z_{2}^{2}$.

3.1.Theorem. For some integers $m, t \geq 2$, let $\varepsilon^{(m)}: E^{(m)} \longrightarrow \mathrm{M}_{t}(\Omega)$ be a CTrepresentation of $E^{(m)}$ over a commutative $K$-algebra $\Omega$. Then the assignments

$$
\begin{gathered}
1 \longmapsto\left[\begin{array}{cc}
I_{t} & 0 \\
0 & I_{t}
\end{array}\right], v_{i} \longmapsto\left[\begin{array}{cc}
\varepsilon^{(m)}\left(v_{i}\right) & 0 \\
0 & -\varepsilon^{(m)}\left(v_{i}\right)
\end{array}\right] \text { for } 1 \leq i \leq m, \\
\text { and } v_{m+1} \longmapsto\left[\begin{array}{cc}
0 & \widehat{z} I_{t} \\
\widehat{z} I_{t} & 0
\end{array}\right] \text { (with } \widehat{z}=z+\left(z^{2}\right) \text { in } \Omega[z] /\left(z^{2}\right) \text { ) }
\end{gathered}
$$

define a CT-representation $\varepsilon^{(m+1)}: E^{(m+1)} \longrightarrow \mathrm{M}_{2 t}\left(\Omega[z] /\left(z^{2}\right)\right)$.

Proof. Any element of $E^{(m+1)}$ can be uniquely written as $g+h v_{m+1}$, where

$$
g=\sum_{1 \leq i_{1}<\ldots<i_{k} \leq m} c_{i_{1}, \ldots, i_{k}} v_{i_{1}} \cdots v_{i_{k}} \text { and } h=\sum_{1 \leq j_{1}<\ldots<j_{l} \leq m} d_{j_{1}, \ldots, j_{l}} v_{j_{1}} \cdots v_{j_{l}}
$$

are in $E^{(m)}$ with $c_{i_{1}, \ldots, i_{k}}, d_{j_{1}, \ldots, j_{l}} \in K$. For $1 \leq i_{1}<\cdots<i_{k} \leq m$ and $1 \leq j_{1}<$ $\cdots<j_{l} \leq m$, our assignment gives

$$
v_{i_{1}} \cdots v_{i_{k}} \longmapsto\left[\begin{array}{cc}
\varepsilon^{(m)}\left(v_{i_{1}} \cdots v_{i_{k}}\right) & 0 \\
0 & (-1)^{k} \varepsilon^{(m)}\left(v_{i_{1}} \cdots v_{i_{k}}\right)
\end{array}\right]
$$

and

Thus

$$
v_{j_{1}} \cdots v_{j_{l}} v_{m+1} \longmapsto\left[\begin{array}{cc}
0 & \varepsilon^{(m)}\left(v_{j_{1}} \cdots v_{j_{l}}\right) \widehat{z} \\
(-1)^{l} \varepsilon^{(m)}\left(v_{j_{1}} \cdots v_{j_{l}}\right) \widehat{z} & 0
\end{array}\right] .
$$

$$
\varepsilon^{(m+1)}\left(g+h v_{m+1}\right)=\left[\begin{array}{cc}
\varepsilon^{(m)}\left(g_{0}+g_{1}\right) & \varepsilon^{(m)}\left(h_{0}+h_{1}\right) \widehat{z} \\
\varepsilon^{(m)}\left(h_{0}-h_{1}\right) \widehat{z} & \varepsilon^{(m)}\left(g_{0}-g_{1}\right)
\end{array}\right],
$$

where $g=g_{0}+g_{1}$ and $h=h_{0}+h_{1}$ are the unique presentations as sums of an even and an odd element (with respect to the natural $\mathbb{Z}_{2}$-grading $E^{(m)}=E_{0}^{(m)} \oplus E_{1}^{(m)}$ ). Straightforward verification shows that $\varepsilon^{(m+1)}: E^{(m+1)} \longrightarrow \mathrm{M}_{2 t}\left(\Omega[z] /\left(z^{2}\right)\right)$ is an injective homomorphism of $K$-algebras. In view of

$$
\operatorname{tr}\left(\varepsilon^{(m+1)}\left(g+h v_{m+1}\right)\right)=\operatorname{tr}\left(\varepsilon^{(m)}\left(g_{0}+g_{1}\right)\right)+\operatorname{tr}\left(\varepsilon^{(m)}\left(g_{0}-g_{1}\right)\right) \in K,
$$

we deduce that $\varepsilon^{(m+1)}$ is a CT-representation of $E^{(m+1)}$.

3.2. Corollary. For any integer $m \geq 2$ there exists a CT-representation

$$
\varepsilon^{(m)}: E^{(m)} \longrightarrow \mathrm{M}_{2^{m-1}}\left(K\left[z_{1}, \ldots, z_{m}\right] /\left(z_{1}^{2}, \ldots, z_{m}^{2}\right)\right)
$$

of $E^{(m)}$, where $\left(z_{1}^{2}, \ldots, z_{m}^{2}\right)$ is the ideal of $K\left[z_{1}, \ldots, z_{m}\right]$ generated by the monomials $z_{1}^{2}, \ldots, z_{m}^{2}$. 
Proof. Starting from $\varepsilon^{(2)}: E^{(2)} \longrightarrow \mathrm{M}_{2}\left(K\left[z_{1}, z_{2}\right] /\left(z_{1}^{2}, z_{2}^{2}\right)\right)$ and using

$$
\left(K\left[z_{1}, \ldots, z_{m}\right] /\left(z_{1}^{2}, \ldots, z_{m}^{2}\right)\right)[z] /\left(z^{2}\right) \cong K\left[z_{1}, \ldots, z_{m}, z_{m+1}\right] /\left(z_{1}^{2}, \ldots, z_{m}^{2}, z_{m+1}^{2}\right),
$$

iteration of the construction in Theorem 3.1 gives the desired CT-representation.

3.3.Proposition. If $\varepsilon: E^{(m)} \longrightarrow \mathrm{M}_{t}(\Omega)$ is a (not necessarily $C T$-) representation of $E^{(m)}$ for some integers $m, t \geq 2$ over a commutative $K$-algebra $\Omega$, then $m \leq$ $2 t-1$.

Proof. Let $S_{m}\left(x_{1}, \ldots, x_{m}\right)=\sum_{\pi \in \operatorname{Sym}(m)} \operatorname{sgn}(\pi) x_{\pi(1)} \cdots x_{\pi(m)}$ be the standard polynomial in $K\left\langle x_{1}, \ldots, x_{m}\right\rangle$. The well-known Amitsur-Levitzki theorem (see [R]) asserts that $S_{2 t}=0$ is a polynomial identity on $\mathrm{M}_{t}(\Omega)$. The existence of the embedding $\varepsilon$ ensures that $S_{2 t}=0$ is also an identity on $E^{(m)}$. On the other hand

$$
S_{m}\left(v_{1}, \ldots, v_{m}\right)=m ! v_{1} \cdots v_{m} \neq 0
$$

shows that $S_{m}=0$ is not a polynomial identity on $E^{(m)}$. If $2 t \leq m$, then $S_{m}=0$ follows from $S_{2 t}=0$ (in any algebra). Thus we have $2 t \nless m$.

3.4.Theorem("Cayley-Hamilton"). If $A \in \mathrm{M}_{n}\left(E^{(m)}\right)$ is an $n \times n$ matrix, then $A$ satisfies an identity of the form

$$
A^{2^{m-1} n}+c_{1} A^{\left(2^{m-1} n\right)-1}+\cdots+c_{\left(2^{m-1} n\right)-1} A+c_{2^{m-1} n} I_{n}=0,
$$

where $c_{i} \in K, 1 \leq i \leq 2^{m-1} n$.

Proof. A combination of Theorem 2.1 and Corollary 3.2 gives the identity.

3.5.Remark. In particular, we know the following about embeddability of $E^{(3)}$ into matrix algebras. By Corollary $3.2, E^{(3)}$ admits a CT-embedding into a $4 \times 4$ matrix algebra. On the other hand, it has no CT-embedding into any $2 \times 2$ matrix algebra. The simple reason is that for $c_{1}, c_{2} \in K$, the $2 \times 2$ Cayley-Hamilton identity

$$
\left(v_{1}+v_{2} v_{3}\right)^{2}+c_{1}\left(v_{1}+v_{2} v_{3}\right)+c_{2}=0
$$

never holds in $E^{(3)}$, although such an identity should hold in $E^{(3)}$ by Theorem 2.1 if it CT-embeds into a $2 \times 2$ matrix algebra. We could not decide whether $E^{(3)}$ has a CT-embedding into a $3 \times 3$ matrix algebra.

For $m=3$ and $t=2$ the inequality $m \leq 2 t-1$ in Proposition 3.3 becomes equality, thus Proposition 3.3 does not contradict the existence of a non CT-representation $\varepsilon: E^{(3)} \longrightarrow \mathrm{M}_{2}(\Omega)$. Indeed, the assignments

$$
1 \longmapsto\left[\begin{array}{cc}
1 & 0 \\
0 & 1
\end{array}\right], v_{1} \longmapsto\left[\begin{array}{cc}
0 & x \\
x & 0
\end{array}\right], v_{2} \longmapsto\left[\begin{array}{cc}
y & 2 y \\
-2 y & -y
\end{array}\right], v_{3} \longmapsto\left[\begin{array}{cc}
-2 z & -z \\
z & 2 z
\end{array}\right]
$$

define a $K$-embedding $E^{(3)} \longrightarrow \mathrm{M}_{2}\left(K[x, y, z] /\left(x^{2}, y^{2}, z^{2}\right)\right)$. Since

$$
v_{1} v_{2} v_{3} \longmapsto\left[\begin{array}{cc}
3 x y z & 0 \\
0 & 3 x y z
\end{array}\right],
$$

this embedding is not a CT-representation. 
3.6.Remark. Any matrix $A \in \mathrm{M}_{n}(E)$ (here $\operatorname{char}(K)=0$ is essential) satisfies left and right Cayley-Hamilton identities of the form

$$
\begin{aligned}
& A^{n^{2}}+g_{1} A^{n^{2}-1}+\cdots+g_{n^{2}-1} A+g_{n^{2}} I_{n}=0, \\
& A^{n^{2}}+A^{n^{2}-1} h_{1}+\cdots+A h_{n^{2}-1}+I_{n} h_{n^{2}}=0,
\end{aligned}
$$

and a central Cayley-Hamilton identity of the form

$$
A^{2 n^{2}}+u_{1} A^{2 n^{2}-1}+\cdots+u_{2 n^{2}-1} A+u_{2 n^{2}} I_{n}=0,
$$

where $g_{i}, h_{i} \in E, 1 \leq i \leq n^{2}$ and $u_{j} \in E_{0}, 1 \leq j \leq 2 n^{2}$ (see [Sz1]).

3.7.Theorem. The standard identity $S_{2^{m} n}=0$ of degree $2^{m} n$ is a polynomial identity on $\mathrm{M}_{n}\left(E^{(m)}\right)$.

Proof. Since the natural extension

$$
\left(\varepsilon^{(m)}\right)_{n}: \mathrm{M}_{n}\left(E^{(m)}\right) \longrightarrow \mathrm{M}_{n}\left(\mathrm{M}_{2^{m-1}}\left(K\left[z_{1}, \ldots, z_{m}\right] /\left(z_{1}^{2}, \ldots, z_{m}^{2}\right)\right)\right)
$$

of $\varepsilon^{(m)}$ (in Corollary 3.2) is a $K$-embedding and

$$
\mathrm{M}_{n}\left(\mathrm{M}_{2^{m-1}}\left(K\left[z_{1}, \ldots, z_{m}\right] /\left(z_{1}^{2}, \ldots, z_{m}^{2}\right)\right)\right) \cong \mathrm{M}_{2^{m-1} n}\left(K\left[z_{1}, \ldots, z_{m}\right] /\left(z_{1}^{2}, \ldots, z_{m}^{2}\right)\right)
$$

satisfies $S_{2^{m} n}=0$ by the Amitsur-Levitzki theorem, the proof is complete.

If $n=1$, then Theorems 3.4 and 3.7 are far from being sharp.

3.8.Remark. Using Theorem 5.5 of Domokos [D] and the fact that $S_{m+2}=0$ is an identity on $E^{(m)}$, we obtain that $S_{(m+1) n^{2}+1}=0$ is an identity on $\mathrm{M}_{n}\left(E^{(m)}\right)$.

The left regular representation of $E^{(m)}$ is an embedding

$$
\lambda^{(m)}: E^{(m)} \longrightarrow \operatorname{End}_{K}\left(E^{(m)}\right) \cong \mathrm{M}_{2^{m}}(K)
$$

of $K$-algebras, where $\operatorname{End}_{K}\left(E^{(m)}\right)$ is the algebra of all $K$-linear maps of the $2^{m}$ dimensional vector space ${ }_{K} E^{(m)}$ and $\lambda^{(m)}(g): E^{(m)} \longrightarrow E^{(m)}$ is the left multiplication by $g \in E^{(m)}$. The size of the matrix algebra is $2^{m-1}$ in Corollary 3.2 , half of the size $2^{m}$ provided by the above left regular representation. On the other hand, the base field $K$ is replaced by a much bigger base ring ( $K$-algebra) in Corollary 3.2. Since we cannot derive Theorems 3.4 and 3.7 from the regular representation, our half sized embedding is really better in many ways than the regular representation.

If we keep $K$ as the base field, then the next theorem gives a lower bound for the matrix size of any possible embedding of $E^{(m)}$.

3.9.Theorem. If $\lambda: E^{(m)} \longrightarrow \mathrm{M}_{t}(K)$ is an embedding of $K$-algebras, then $3 \cdot 2^{m-2}=2^{m-1}+2^{m-2} \leq\left\lfloor\frac{t^{2}}{4}\right\rfloor+1$.

Proof. Consider the $K$-subalgebra $E_{0}^{(m)}\left[v_{m}\right]$ of $E^{(m)}$ generated by the centre $E_{0}^{(m)}$ and the generator $v_{m}$. Clearly, $E_{0}^{(m)}\left[v_{m}\right]$ is commutative, and any element of $E_{0}^{(m)}\left[v_{m}\right]$ is of the form $g_{0}+h_{0} v_{m}$, where $g_{0} \in E_{0}^{(m)}$ and $h_{0} \in E_{0}^{(m-1)}$. It follows that $\operatorname{dim}_{K} E_{0}^{(m)}\left[v_{m}\right]=2^{m-1}+2^{m-2}$. Since $\lambda\left(E_{0}^{(m)}\left[v_{m}\right]\right) \subseteq \mathrm{M}_{t}(K)$ is a commutative subalgebra of $\mathrm{M}_{t}(K)$, we can apply Schur's inequality (see $[\mathrm{M}]$ ):

$$
2^{m-1}+2^{m-2}=\operatorname{dim}_{K} E_{0}^{(m)}\left[v_{m}\right]=\operatorname{dim}_{K} \lambda\left(E_{0}^{(m)}\left[v_{m}\right]\right) \leq\left\lfloor\frac{t^{2}}{4}\right\rfloor+1 .
$$


3.10.Remark. Since M. Domokos and M. Zubor recently proved (see [DZ]) that $\operatorname{dim}_{K} C \leq 3 \cdot 2^{2 r-2}$ for any commutative $K$-subalgebra $C \subseteq E^{(2 r)}$ of $E^{(2 r)}$, the argument in the above proof cannot be improved if $m=2 r$ is even.

\section{SKEW POLYNOMIAL RINGS AND EMBEDDINGS}

For a ring ( $K$-algebra) endomorphism $\sigma: R \longrightarrow R$, consider the skew polynomial ring (K-algebra) $R[w, \sigma]$ in the skew indeterminate $w$. The elements of $R[w, \sigma]$ are left polynomials of the form $f(w)=a_{0}+a_{1} w+\cdots+a_{k} w^{k}$ with $a_{0}, a_{1}, \ldots, a_{k} \in R$. Besides the obvious addition, we have the following multiplication rule in $R[w, \sigma]$ : $w r=\sigma(r) w$ for all $r \in R$ and

$$
\left(a_{0}+a_{1} w+\cdots+a_{k} w^{k}\right)\left(b_{0}+b_{1} w+\cdots+b_{l} w^{l}\right)=c_{0}+c_{1} w+\cdots+c_{k+l} w^{k+l},
$$

where

$$
c_{m}=\sum_{i+j=m, i \geq 0, j \geq 0} a_{i} \sigma^{i}\left(b_{j}\right)
$$

If $\sigma$ is an involution, then $w^{2}$ is a central element of $R[w, \sigma]$ : we have $\sigma(\sigma(r))=r$ and $w^{2} r=w \sigma(r) w=\sigma(\sigma(r)) w^{2}=r w^{2}$ for all $r \in R$, moreover $w^{2}$ commutes with the powers of $w$. Thus the ideal $\left(w^{2}\right)$ of $R[w, \sigma]$ generated by $w^{2}$ can be written as $\left(w^{2}\right)=R[w, \sigma] w^{2}=w^{2} R[w, \sigma]$. Consider the factor ring (K-algebra) $R[w, \sigma] /\left(w^{2}\right)$, then for any element $f(w) \in R[w, \sigma]$ there exists exactly one left polynomial of the form $r+s w \in R[w, \sigma]$ in the residue class $f(w)+\left(w^{2}\right)$. Hence the elements of $R[w, \sigma] /\left(w^{2}\right)$ can be represented by linear left polynomials with coefficients in $R$, and the multiplication in $R[w, \sigma] /\left(w^{2}\right)$ is the following:

$$
(r+s w)(p+q w)=r p+(r q+s \sigma(p)) w,
$$

where $r, s, p, q \in R$.

If $R=E=E_{0} \oplus E_{1}$ (or $R=E^{(m)}=E_{0}^{(m)} \oplus E_{1}^{(m)}$ ) is the natural $\mathbb{Z}_{2}$-grading, then $\sigma(g)=\sigma\left(g_{0}+g_{1}\right)=g_{0}-g_{1}$ defines a natural involution (here $g=g_{0}+g_{1}$ is the unique presentation as a sum of an even and an odd element). It is easy to see that $E[w, \sigma] /\left(w^{2}\right) \cong E$ and $E^{(m)}[w, \sigma] /\left(w^{2}\right) \cong E^{(m+1)}$ as $K$-algebras.

We note that the idea of considering $R[w, \sigma] /\left(w^{2}\right)$ comes from [SSz].

4.1.Theorem("Fundamental Embedding"). For an involution $\sigma: R \longrightarrow R$, putting

$$
\mu\left(r+s w+\left(w^{2}\right)\right)=\left[\begin{array}{cc}
r+\left(z^{2}\right) & s z+\left(z^{2}\right) \\
\sigma(s) z+\left(z^{2}\right) & \sigma(r)+\left(z^{2}\right)
\end{array}\right]
$$

(with $r, s \in R$ ) gives a K-embedding $\mu: R[w, \sigma] /\left(w^{2}\right) \longrightarrow \mathrm{M}_{2}\left(R[z] /\left(z^{2}\right)\right.$ ).

Proof. We only have to prove the multiplicative property of $\mu$ :

$$
\begin{gathered}
\mu\left(\left(r+s w+\left(w^{2}\right)\right)\left(p+q w+\left(w^{2}\right)\right)\right)=\mu\left(r p+(r q+s \sigma(p)) w+\left(w^{2}\right)\right)= \\
{\left[\begin{array}{cc}
r p+\left(z^{2}\right) & (r q+s \sigma(p)) z+\left(z^{2}\right) \\
\sigma(r q+s \sigma(p)) z+\left(z^{2}\right) & \sigma(r p)+\left(z^{2}\right)
\end{array}\right]=} \\
{\left[\begin{array}{cc}
r+\left(z^{2}\right) & s z+\left(z^{2}\right) \\
\sigma(s) z+\left(z^{2}\right) & \sigma(r)+\left(z^{2}\right)
\end{array}\right] \cdot\left[\begin{array}{cc}
p+\left(z^{2}\right) & q z+\left(z^{2}\right) \\
\sigma(q) z+\left(z^{2}\right) & \sigma(p)+\left(z^{2}\right)
\end{array}\right]=} \\
\mu\left(r+s w+\left(w^{2}\right)\right) \mu\left(p+q w+\left(w^{2}\right)\right) .
\end{gathered}
$$

The following is a broad generalization of Theorem 3.1. 
4.2. Theorem. Let $\varepsilon: R \longrightarrow \mathrm{M}_{t}(\Omega)$ be a CT-representation of $R$ over a commutative $K$-algebra $\Omega$ for some integer $t \geq 2$. If $\sigma: R \longrightarrow R$ is an involution, then there exists an induced CT-representation $\varepsilon^{*}: R[w, \sigma] /\left(w^{2}\right) \longrightarrow \mathrm{M}_{2 t}\left(\Omega[z] /\left(z^{2}\right)\right)$ of the factor $R[w, \sigma] /\left(w^{2}\right)$.

Proof. Consider the natural extension

$$
\bar{\varepsilon}_{2}: \mathrm{M}_{2}\left(R[z] /\left(z^{2}\right)\right) \longrightarrow \mathrm{M}_{2}\left(\left(\mathrm{M}_{t}(\Omega)[z]\right) /\left(z^{2}\right)\right) \cong \mathrm{M}_{2 t}\left(\Omega[z] /\left(z^{2}\right)\right)
$$

of $\bar{\varepsilon}: R[z] /\left(z^{2}\right) \longrightarrow\left(\mathrm{M}_{t}(\Omega)[z]\right) /\left(z^{2}\right)$, where $\bar{\varepsilon}\left(r+s z+\left(z^{2}\right)\right)=\varepsilon(r)+\varepsilon(s) z+\left(z^{2}\right)$ for $r, s \in R$. Using the map $\mu: R[w, \sigma] /\left(w^{2}\right) \longrightarrow \mathrm{M}_{2}\left(R[z] /\left(z^{2}\right)\right)$ in Theorem 4.1, the composition $\varepsilon^{*}=\bar{\varepsilon}_{2} \circ \mu$ gives the induced CT-representation. Indeed, $\operatorname{tr}\left(\varepsilon^{*}\left(r+s w+\left(w^{2}\right)\right)\right)=\left(\operatorname{tr}(\varepsilon(r))+\left(z^{2}\right)\right)+\left(\operatorname{tr}(\varepsilon(\sigma(r)))+\left(z^{2}\right)\right) \in K+\left(z^{2}\right)$.

Let $\sigma: R \longrightarrow R$ be a ( $K$-algebra) endomorphism with $\sigma^{t}=1$ (such a $\sigma$ is an automorphism). Now $w^{t}$ is a central element of $R[w, \sigma]$ and the ideal $\left(w^{t}\right)$ of $R[w, \sigma]$ can be written as $\left(w^{t}\right)=R[w, \sigma] w^{t}=w^{t} R[w, \sigma]$. We close this section by mentioning the following generalization of Theorem 4.1 (see [Sz2]), which seems to have applications in Galois theory.

4.3.Theorem. For an endomorphism $\sigma: R \longrightarrow R$ with $\sigma^{t}=1$, putting

$$
\mu\left(r_{0}+r_{1} w+\cdots+r_{t-1} w^{t-1}+\left(w^{t}\right)\right)=\left[\sigma^{i-1}\left(r_{j-i}\right) z^{j-i}+\left(z^{t}\right)\right]_{t \times t}
$$

gives an embedding $\mu: R[w, \sigma] /\left(w^{t}\right) \longrightarrow \mathrm{M}_{t}\left(R[z] /\left(z^{t}\right)\right)$, where the difference $j-i \in$ $\{0,1, \ldots, t-1\}$ is taken modulo $t$, and the element $\sigma^{i-1}\left(r_{j-i}\right) z^{j-i}+\left(z^{t}\right)$ of the factor $R[z] /\left(z^{t}\right)$ is in the $(i, j)$ position of the $t \times t$ matrix $\left[\sigma^{i-1}\left(r_{j-i}\right) z^{j-i}+\left(z^{t}\right)\right]_{t \times t}$. The trace of $\left[\sigma^{i-1}\left(r_{j-i}\right) z^{j-i}+\left(z^{t}\right)\right]_{t \times t}$ is in $R^{\sigma}+\left(z^{t}\right)$, where $R^{\sigma}=\{r \in R \mid \sigma(r)=r\}$ is the fixed ring of $\sigma$.

\section{REFERENCES}

[D] M. Domokos, Eulerian polynomial identities and algebras satisfying a standard identity, J. Algebra 169(3) (1994), 913-928.

[DZ] M. Domokos and M. Zubor, Commutative subalgebras of the Grassmann algebra, arXiv:1403.2916

[K] A. R. Kemer, Ideals of Identities of Associative Algebras, Translations of Math. Monographs, Vol. 87 (1991), AMS, Providence, Rhode Island.

[M] M. Mirzakhani, A simple proof of a theorem of Schur, Amer. Math. Monthly 105(3) (1998), 260-262.

[R] L.H. Rowen, Polynomial identities in ring theory, Academic Press, New York, 1980.

[SSz] S. Sehgal and J. Szigeti, Matrices over centrally $\mathbb{Z}_{2}$-graded rings, Beitr. Algebra Geom. 43(2) (2002), 399-406.

[Sz1] J. Szigeti, New determinants and the Cayley-Hamilton theorem for matrices over Lie nilpotent rings, Proc. Amer. Math. Soc. 125(8) (1997), 2245-2254.

[Sz2] J. Szigeti, Embedding truncated skew polynomial rings into matrix rings and embedding of a ring into $2 \times 2$ supermatrices, arXiv:1307.1783 
[SzvW] J. Szigeti and L. van Wyk, Determinants for $n \times n$ matrices and the symmetric Newton formula in the $3 \times 3$ case, Linear and Multilinear Algebra, Vol. 62, No.8 (2014), 1076-1090.

Alfréd Rényi Institute of Mathematics, Hungarian Academy of Sciences, 1364 BuDAPest, Pf. 127, Hungary

E-mail address: marki.laszlo@renyi.mta.hu

Department of Mathematics and Applied Mathematics, University of the Free State, PO Box 339, Bloemfontein 9300, South Africa

E-mail address: MeyerJH@ufs.ac.za

Institute of Mathematics, University of Miskold, 3515 Miskold-Egyetemváros, HunGARY

E-mail address: matjeno@uni-miskolc.hu

Department of Mathematical Sciences, Stellenbosch University P/Bag X1 Matieland 7602, Stellenbosch, South Africa

E-mail address: LvW@sun.ac.za 